\title{
Cell interactions and gene interactions in peripheral neurogenesis
}

\author{
Alain Ghysen, ${ }^{1}$ Christine Dambly-Chaudière,, ${ }^{1}$ Lily Y. Jan, ${ }^{2}$ and Yuh-Nung Jan ${ }^{2}$ \\ ${ }^{1}$ Laboratoires de Neurobiologie et de Génétique, Université Libre de Bruxelles, 1640 Rhode-St-Genèse, Belgium; ${ }^{2}$ Howard \\ Hughes Medical Institute and the Departments of Physiology and Biochemistry, University of California at San Francisco, \\ San Francisco, California 94143 USA
}

The formidable complexity inherent in the development of an organism is the outcome of the interlacing or concatenation of a large number of discrete steps. Genetic analyses have revealed that each of these steps is a relatively simple developmental event resulting from the activity of a small battery of genes, often interacting with one another. This, at least, is the picture that emerges from work on developmental events as diverse as segmentation, dorsoventral organization, and segment determination in the fly embryo (Lewis 1978; Nüsslein Volhard and Wieschaus 1980; for review, see St. Johnston and Nüsslein-Volhard 1992). The expression of the appropriate battery of genes at the appropriate time and place is obviously of crucial importance. Thus, the question is How is this multiplicity of parallel programs kept in register?

In the case of segmentation (spatial subdivision of the embryo|, analysis has revealed that a succession of steps leads from simple anteroposterior heterogeneities in the distribution of a few key factors present in the oocyte to the reproducible establishment of 14 consecutive metameres shortly after cellularization of the embryo. At a late stage of this process, boundaries are set up between parasegmental metameres (for review, see Lawrence 1992). Later on, the different metameres will adopt their individual identity and develop independently of each other. For example, mutations that lead to the absence of several segments have little effect on the development of those segments that remain. By the end of the segmentation process, therefore, the embryonic ectoderm is effectively partitioned into independent developmental units.

By analogy to segmentation, does the definition of specific cell fates within a particular tissue involve a progressive subdivision of the tissue until each tiny developmental unit contains only one pattern element, at the limit, down to developmental units that would be made of only one cell? The development of sense organs is a case in point. Here, the end result is the differentiation of specific cells at specific positions of the epidermis to generate recognizable sense organs.

The formation of each sense organ, however, is not the end result of a cascade of compartments within compartments within compartments. Rather, cells at particular locations of the tissue are instructed to adopt a particular fate. How is this process programed by the genome? Here, again, it appears that the entire process results from the concatenation of a number of different steps, each of which depends on the function of a small battery of genes. In this review we will concentrate on the early part of the process-the singling out of the precursor cells-using the sensory bristles of the adult Drosophila as a model system (Fig. 1). We will discuss how independent developmental units are transiently established for the determination of each bristle and how the three sets of genes that are involved in this process work in concert to single out the precursor cells.

\section{Three key genes: two too many?}

Each sense organ originates as a single precursor cell called a sensory mother cell (SMC). What is the mechanism for the singling out of SMCs from epithelial cells?

Mutations that alter the formation of SMCs show either of two contrasting phenotypes: Some reduce the number of SMCs, and others increase it. The analysis of mutant phenotypes can give us cues about the normal function of the wild-type gene. The first step in this analysis is to distinguish between loss-of-function (LOF) mutations, which inactivate the gene, and gain-of-function (GOF) mutations, which lead to its overexpression or ectopic expression. The rationale of this analysis is that LOF mutations show the defect due to inactivation of the gene and, thus, inform us about the requirements of the gene. On the other hand, GOF mutations show the alteration due to an excess of gene activity and tell us what the gene is sufficient for (i.e., provided all other factors are present). Thus, if we can know both the LOF and GOF phenotypes of a given gene, we will then have a good idea of the function of that gene. For example, if the LOF phenotype of a given gene results in the absence of structure A while the GOF phenotype results in extra copies of structure A being formed, then one can conclude that the gene is responsible for the decision to develop structure A. This principle of functional analysis was first established by E.B. Lewis in demonstrating that the homeotic gene Ultrabithorax is responsible for the acquisition of the proper segmental identity by metatho- 
Figure 1. The precursor (SMC) of a sense organ is singled out from the epidermis in a process that involves a concatenation of different steps. At a stage when the entire imaginal disc contains several thousand cells, an autonomous unit emerges for the determination of a bristle precursor. First, the independent control of $e m c$ and $s c$ expression, probably by regionally distributed transcriptional regulators, defines the refractory regions and the proneural clusters, respectively. Second, those cells with sufficient levels of the active form of the sc protein, which is not sequestered by the emc protein, acquire competence to become neural precursors and begin to express the inhibitory signal, the $\mathrm{Dl}$ protein. The $\mathrm{Dl}$ protein binds to the $\mathrm{N}$ protein of neighboring cells and activates it. The expression of $D I$ in the cluster of competent cells therefore results in the activation of the $\mathrm{N}$ protein (and consequently in the inactivation of the sc protein) in a larger cluster-the inhibitory field. Thus, although $\mathrm{N}$ is ubiquitously expressed, only those cells in the inhibitory field have $\mathrm{N}$ in the activated form. The inactivation of $\mathrm{sc}$ will be felt primarily in the cells that express sc at a moderate or low level. Each time one of these cells drops off the competition, its inhibitory effect on the remaining cells will disappear, and the remaining cells will therefore become more and more competent until only two or three cells are left that contain a relatively high level of sc activity (pre-precursors). Finally one of the pre-precursors will emerge as maximally competent while all of its neighbors will be maximally inhibited. At this point, the remaining competent cell is free of activated $\mathrm{N}$, because the other cells have lost the ability to produce $\mathrm{Dl}_{\text {; }}$ its rate of expression of $s c$ jumps to the high level typical of the precursors, presumably by relying on the autocatalytic control of $s c$ expression. Except for the activation of $D l$ expression by sc, other interactions are probably at the post-transcriptional level. The inhibition of $s c$ by emc is the result of the formation of inactive heterodimers, and the activation of $N$ by

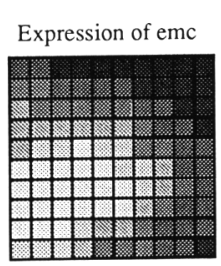

refractory regions

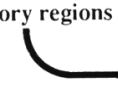

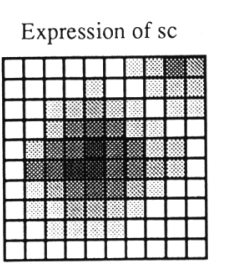

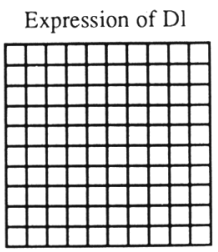

Expression of $\mathrm{N}$

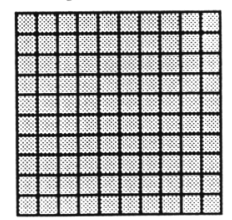

proneural cluster

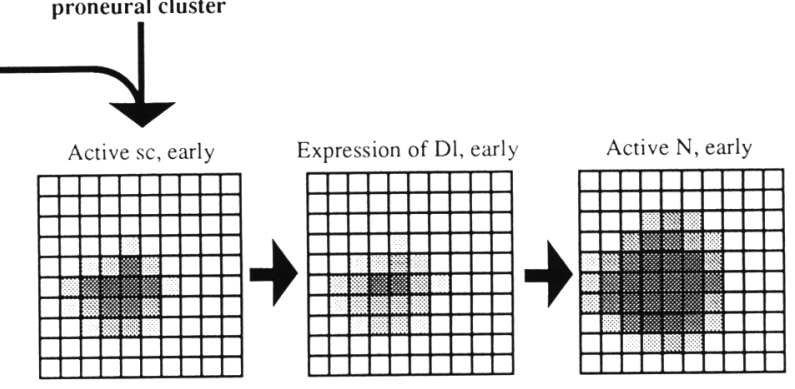

inhibitory field

competence
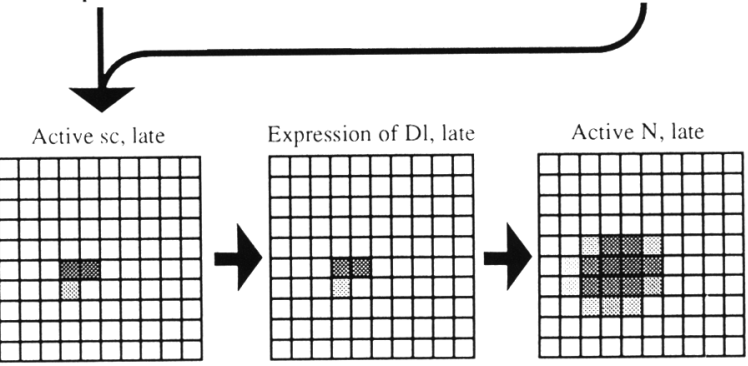

pre-precursors

inhibitory field
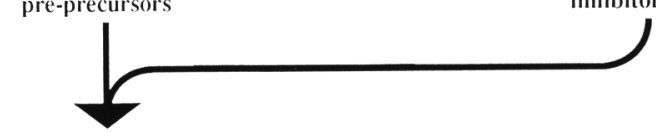

Active sc, final

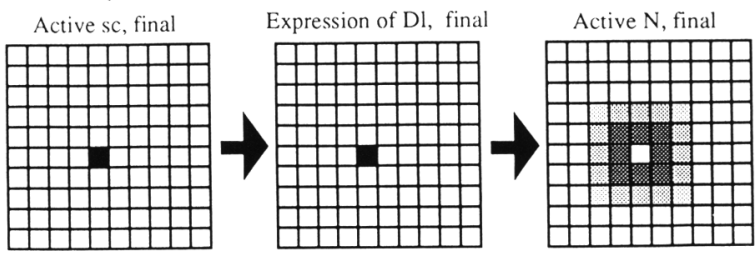

Active $\mathrm{N}$, final

$D I$ is the result of a direct interaction between the extracellular domains of the two proteins. The inactivation of sc by the activated $\mathrm{N}$ protein might be mediated by one or more of several mechanisms that might affect the activity of the protein directly, or might act at the transcriptional level, for example, by preventing the autocatalytic control of $s c$ to be turned on.

racic cells (Lewis 1978). Since then, the method has been widely used both in flies and in other organisms to assess the role of other developmentally important genes.

In the particular case of the sense organs, this analysis was performed for three genes: scute (sc), extramacrochaetae (emc), and Notch $(N)$. The genetic evidence strongly suggests that each of these three genes is the key gene responsible for the singling out of individual ectodermal cells as SMCs. How can three different genes be both necessary and sufficient for a single decision?

As discussed below, at least two of these genes, $s c$ and $N$, each belongs to a set of genes that appear to exert similar functions. Thus, $s c$ belongs to the set of proneural genes that endow clusters of ectodermal cells /the proneural clusters) with the potential to form neural precursors, whereas $N$ belongs to the set of neurogenic genes that appear to limit the number of precursors that are formed within a proneural cluster. In this review we will concentrate on $s c$, emc, and $N$ as representatives of three different sets of genes; however, it should be kept in mind that additional genes of these sets are likely to be both necessary and sufficient for the decision of an ectodermal cell to form a SMC.

sc

The gene sc belongs to a complex locus, the achaetescute gene complex (AS-C), which includes four structurally and functionally related genes: achaete $(a c), s c$, lethal of scute (1sc), and asense (ase) (Garcia-Bellido 1978; Garcia-Bellido and Santamaria 1978; Campuzano 
et al. 1985; Dambly-Chaudière and Ghysen 1987). All four AS-C gene products probably act as transcriptional regulators and contain a motif called helix-loop-helix (HLH), which mediates the formation of homo- or heterodimers (Villares and Cabrera 1987; Alonso and Cabrera 1988; Gonzalez et al. 1989; Murre et al. 1989). Preceding the HLH motif is a basic DNA-binding domain. Heterodimers with the product of the daughterless (da) gene, which is a ubiquitous HLH cofactor, are probably the predominant active forms (Caudy et al. 1988; Cabrera and Alonso 1991).

LOF mutations in any of the AS-C genes result in the loss of neural elements. Which elements are lost depends on which gene is affected: In particular, the inactivation of the gene $s c$ eliminates most of the large mechanosensory bristles of the adult. Therefore, the function of $s c$ is required for the development of these bristles.

GOF mutations of $s c$ have also been identified; these mutations result in the appearance of supernumerary sense organs (Garcia-Alonso and Garcia-Bellido 1986; Barcells et al. 1988). These results suggest that $s c$ is a key gene involved in the decision to form a SMC.

Analysis of the distribution of $s c$ transcripts and proteins has revealed that $s c$ is expressed in the imaginal discs before the singling out of the SMCs. At each location where a bristle will form, $s c$ is initially expressed in a cluster of cells; it then accumulates at higher levels in one cell of the cluster-the SMC (Cubas et al. 1991; Skeath and Carroll 1991). In some LOF mutations of $s c$, transcripts fail to accumulate in some of the clusters and the sense organs at those locations do not form. Conversely, the presence of GOF mutations in $s c$ leads to the accumulation of transcripts and protein in all or most cells of the tissue, resulting in the formation of supernumerary bristles (Campuzano et al. 1986; Ruiz-Gomez and Modolell 1987; Balcells et al. 1988).

These results suggest that the expression of $s c$ is a prerequisite for the formation of a SMC and lead to the conclusion that $s c$ (and other genes of the AS-C) provides the competence to become a SMC to groups of cells. For this reason the AS-C genes were called proneural (Ghysen and Dambly Chaudière 1989; Romani et al. 1989).

The pattern of expression of the AS-C genes is therefore a crucial element in the formation of the pattern of sense organs. How is this pattern of expression established? On the basis of the analysis of a large number of rearrangements with breakpoints upstream and downstream of the $s c$-coding region, it has been proposed that the formation of this pattern depends on an array of regulatory sites extending over several tens of kilobases downstream of the $s c$-coding region (Ruiz-Gomez and Modolell 1987; Leyns et al. 1989). Each regulatory site would be responsible for the expression of $s c$ in one cluster of cells at a particular position in the imaginal disc, probably as a response to a particular combination of regionally distributed transcriptional regulators.

The identity of the trans-regulators that act at each of these control sites has not yet been addressed in the imaginal discs, where the genetics of positional information is still rudimentary. In the embryo, the tartan-like pattern resulting from the expression of specific combinations of segmentation and dorsoventral genes at different positions of the embryonic ectoderm is at least partly responsible for controlling the local expression of AS-C genes prior to neuroblast segregation (Skeath and Carroll 1992). All of these results are entirely consistent with the idea that the AS-C genes are embedded in an array of regulatory sites, each of which would respond to a particular combination of regionally expressed transcriptional regulators.

It has been demonstrated that at least two of the AS-C genes, $a c$ and $s c$, can trans-activate each other, both in imaginal discs (Martinez and Modolell 1991) and in the embryo (Ruiz-Gomez and Ghysen 1993). This mutual trans-activation of AS-C genes does not appear to fill a particular function in the formation of the SMCs. In the embryo, trans-activation plays no obvious role in setting up the pattern of expression of $a c$. In the disc, the clusters in which $a c$ is expressed owing to trans-activation by $s c$ correspond to sense organs that develop normally in the absence of ac, suggesting that nothing would be changed if $a c$ had not been trans-activated in the corresponding mother cells. Thus, it seems likely that in most cases, trans-activation of AS-C genes by each other simply reflects the self-activation of each gene by its own product and serves no particular purpose. Self-activation, on the other hand, might be instrumental in shifting the level of expression of AS-C from the lower, "competent" level observed in the cells of the proneural clusters, to the higher, "determined" level observed in the SMCs.

\section{emc}

A second gene that has been subjected to the LOF-GOF analysis is emc. LOF mutations of emc were originally identified by virtue of their gene dose titration effect on the AS-C genes (Botas et al. 1982). emc mutations are normally recessive but behave as dominant mutations if the number of copies of the AS-C is increased. Weak LOF mutations of emc are homozygous viable and form supernumerary bristles in the adult located around the sites where the normal bristles form and in the regions that are void of bristles in wild-type flies. More extreme mutations are lethal but can be studied in clones in mosaic flies, where they also result in the development of supernumerary bristles. One mutation localized near $e m c$ has the opposite effect: Some of the normal bristles are absent. The mutation was therefore called Achaetous $(A c h)$. The genetic analysis of Ach demonstrated that this mutation is a GOF allele of emc (Garrell and Modolell 1990). As in the case of $s c$, therefore, we must conclude that $e m c$ is critically involved in the decision not to form a SMC. If emc is absent, too many SMCs are formed, and if it is overexpressed, not enough SMCs develop. How is it that two different genes are both necessary and sufficient for one given process-the decision to form a SMC? And how is it that one of these genes indicates that the key decision is to form a SMC while the other indicates that the key decision is the opposite-not to form a SMC? 
The resolution of this apparent paradox is hinted at by the results of the molecular analysis of emc \{Ellis et al. 1990; Garrell and Modolell 1990). This gene turns out to code for another HLH protein, one in which the basic region adjacent to the $\mathrm{HLH}$ motif is missing. Because this basic region is supposed to mediate DNA recognition, the emc product probably acts in the imaginal discs as a negative regulator of $s c$ by sequestering the $s c$ and/or da protein in the form of inactive sc-emc and/or da-emc heterodimers (Cabrera and Alonso 1991; Van Doren et al. 1991). $s c$ is capable of activating itself as well as other members of the AS-C, and so emc might indirectly down-regulate $s c$ expression as well.

In contrast to $s c$, emc is ubiquitously expressed. Its level of expression, however, is not homogeneous. The regions in which emc is expressed at a higher level are roughly complementary to those in which $s c$ is expressed, so that most of the clusters in which $s c$ is expressed are also regions in which emc is relatively underexpressed (Cubas and Modolell 1992). There does not seem to be any causal relation between the two patterns, however; the distribution of emc transcripts is not affected detectably in $s c$ mutants, and the distribution of $s c$ transcripts is little modified, if at all, in emc mutants. The independent control of emc and $s c$ transcripts provides one mechanism to sharpen the spatial regulation of proneural cluster formation; the level of active sc protein is likely to be reduced in the regions of overlap of emc and $s c$ expression.

The idea that the acquisition of competence depends on a balance between emc and $s c$ helps to clarify several puzzling observations. In particular, the phenotype of GOF mutants in which $s c$ is expressed ubiquitously does not result in the formation of supernumerary sense organs everywhere: Additional bristles are formed only at some locations. Likewise, the ubiquitous expression of $s c$ attributable to the activation of a $h s p-s c$ (heat shock promoter-scute) transgene leads to the formation of additional bristles located at positions similar to those observed in GOF mutants (Rodriguez et al. 1990), suggesting that some regions of the epidermis are refractory to the action of $s c$. This refractoriness can now be explained, at least in part, by the expression of emc at a higher level in these regions. The overexpression of $s c$ in an emc mutant leads to a much more generalized distribution of supernumerary SMCs (Cubas and Modolell 1992).

Depending on the region of the tissue, the expression of emc will contribute to the final pattern of bristles in two different ways. First, the uneven emc expression pattern contributes to the existence of refractory regions; ectopic bristles appear in these regions in LOF emc mutants. Second, as described below, one cell is singled out from a proneural cluster to become a SMC by cell interactions involving direct cell contact. A proneural cluster in the imaginal disc contains $20-30$ cells and may be too large for each cell to be in direct contact with all other cells of the cluster. In the wild type, the partial overlap between the domains of $s c$ expression and the regions in which emc is expressed at a high level will effectively reduce the number of cells in which active sc protein will accumulate. In emc mutants, this reduction will not take place, the range of contact inhibition may be exceeded, and supernumerary bristles may therefore form around normal ones.

The analysis so far suggests that the prime determinant for competence is the local expression of $s c$ under the control of specific position-dependent sites. The level of $s c$ is not sufficient, however, to determine whether a cell will become competent or not: What matters is that there is sufficient $s c$ to overcome sequestering by emc. That is, the balance between emc and $s c$ provides a tuning mechanism that further refines the positional specificity encoded in the sc regulatory elements. Because the acquisition of competence depends on the balance between $s c$ and $e m c$, the reason why manipulating either of the two genes may affect the final decision and, therefore, why the genetic analysis can point to both of them as being a key gene, is obvious.

N

The third gene for which an extensive analysis has been performed is $N$. LOF alleles of $N$ are embryonic lethal. Mutant embryos show a massive hypertrophy of both the central and peripheral nervous systems /CNS and PNS, respectively) at the expense of the epidermis (Poulson 1937). $N$ is a representative of a family of genes that show a similar LOF phenotype and have therefore collectively been called "neurogenic" (Lehmann et al. 1981). In the case of the larval PNS, it has been shown that the hypertrophy of the PNS produced by LOF mutations in the neurogenic genes is the result of the singling out of too many SMCs (Goriely et al. 1991). Because of its lethality, the LOF phenotype is more difficult to assess in the adult. However, a partial decrease in $N$ function already results in the formation of an excess of bristles in the adult, an effect probably resulting from the formation of supernumerary SMCs (Dietrich and Campos-Ortega 1984; Hartenstein and Posakony 1990; Heitzler and Simpson 1991). These results suggest that $N$ is required to restrict the number of cells that will become SMCs.

Another class of mutations localized near the LOF $N$ alleles causes the opposite phenotype: a reduction in the number of sense organs. Here, again, the genetic (Foster 1975; Portin 1975) and molecular (Hartley et al. 1987; Kelley et al. 1987) analyses have revealed that these are GOF alleles of the $N$ gene. So we now have a third gene that seems to be responsible for the decision to form a $\mathrm{SMC}$. How does $N$ fit in with the two key genes that we already have?

The molecular analysis of $N$ revealed that the $N$ product is a membrane protein with a large extracellular domain containing multiple repeats of the EGF motif (Wharton et al. 1985; Kidd et al. 1986). Among the other neurogenic loci, one $(D I)$ codes for another transmembrane protein with a structure similar to that of $N$ (Vässin et al. 1987; Kopczynski et al. 1988); and in the same pathway, a third one Enhancer of split [E(spl)] com- 
prises several genes, one of which codes for putative signal-transducing molecules (Hartley et al. 1988; Klämbt et al. 1989). These observations suggest that at least some of the neurogenic loci act by setting up a system of cell communication that is somehow involved in the determination of the SMCs.

Phenotypic analyses of LOF mutations in the neurogenic loci have suggested that they may be involved in a system of lateral inhibition that would prevent neighboring cells from becoming SMCs (Wigglesworth 1940; Simpson 1990). Lateral inhibition has been invoked previously as the underlying mechanism for the regularity of spacing between bristles and the emergence of new bristles at locations apart from existing bristles during molting in Rhodnius.

How exactly is lateral inhibition achieved? Inhibition was initially proposed to result from competition for some diffusible factor essential for SMC activity. A cell that has become a SMC would use up much of this factor and, by depleting the surrounding cells of this substance, would effectively prevent them from becoming SMCs. Alternatively, the SMC might produce an inhibitory substance that actively prevents the surrounding cells from following the same fate. It now appears that the system used in the adult fly is neither one, but is a third system ensuring that in no case can two neighboring cells become SMCs at the same time-an accident that happens rarely yet could not be prevented by either of the two simple schemes mentioned above.

\section{Mosaic analysis of cell communication}

If $N$ codes for a membrane protein mediating cell-cell communication involved in the process of lateral inhibition, it is important to determine whether $N$ is on the signaling, on the receiving, or on both ends of this communication. This was determined by resorting to mosaic analysis, which makes it possible to juxtapose cells of different genotypes and to examine their respective behaviors. In the fly, this analysis can be performed by irradiating the developing animal with $\mathrm{X}$-rays or by introducing the yeast flip recombinase system into the fly. Occasionally, an event of somatic recombination will make one of the daughter cells homozygous for one chromosome arm (Golic and Linquist 1989). Mitotic recombination can be used to produce clones of cells homozygous for a particular mutation in an otherwise heterozygous animal. This may allow, for example, an analysis of the phenotype of mutations that are lethal for the organism but not for individual cells. Mosaic analysis may also allow one to decide whether the behavior of a cell depends strictly on its own genome or is influenced by the behavior of surrounding cells.

If $N$ is on the receiving end, its inactivation in one cell should make this cell insensitive to lateral inhibition; if it is on the signaling end, it should make this cell unable to inhibit its neighbors but still able to respond to inhibition by its neighbors. If $N$ acts on both ends, its inactivation in one cell will probably affect both the mutant cell and its neighbors. The experiments show that the first answer is correct: Mutant $N$ cells never form epidermis in regions where bristles are normally present (de Celis et al. 1991; Heitzler and Simpson 1991). This result is not attributable to an inability of mutant $N$ cells to differentiate as epidermal cells, however, because such mutant cells can differentiate into epidermal cells in regions where proneural genes $a c$ and $s c$ are not expressed and sense organs never form. Mutant $N$ cells also form epidermis in those regions of $a c$ or $s c$ mutants in which sense organs fail to form owing to the loss of proneural gene function (Heitzler and Simpson 1991).

These results show that a cell must contain an active $N$ gene to be sensitive to the epidermalizing effect of lateral inhibition. The mutant cells, however, do not form sense organs either. This suggests that $N$ is not only required for the singling out of the SMC but also at some later step for the proper development of the sense organ. The analysis of a weak thermosensitive allele has shown that the inactivation of $N$ at the time of the singling out of the SMC results in the segregation of too many mother cells, but the inactivation at a later time results in all the progeny of the SMCs becoming neural (Hartenstein and Posakony 1990). Thus, the final phenotype of clones homozygous for extreme alleles results from the addition of the two effects: First, the mutant $N$ cells become SMCs because they cannot respond to inhibition; and second, all the progeny of these SMCs become neural.

Similar experiments have argued that another member of the same family, Delta $(D I)$, which is also a transmembrane protein with a large extracellular domain containing epidermal growth factor (EGF) repeats, is on the signaling end. Cells that are mutant for a strong $D I$ allele can nevertheless produce epidermis if they are in close contact with wild-type cells at the border of the clone (Heitzler and Simpson 1991).

These results led to the hypothesis that the extracellular domains of the $\mathrm{Dl}$ and $\mathrm{N}$ proteins interact directly. Further evidence for a direct interaction between the $\mathbf{N}$ and $\mathrm{Dl}$ products was later derived from the observed adhesion between cells expressing $N$ and cells expressing DI (Rebay et al. 1991). The adhesion assay, however, which presumably measures the strength of the interactions between the extracellular domains of the $\mathrm{N}$ and $\mathrm{Dl}$ proteins, cannot fully account for the strength of the interaction between specific alleles of $N$ and $D l$. Thus, the function of the N-Dl interaction is not, or not exclusively, to increase adhesion. These observations are consistent with the idea that the products of $N$ and $D I$ not only promote contact between neighboring cells but also mediate intercellular signaling via ligand (Dl)-receptor $(\mathrm{N})$ binding, such that the interaction somehow causes the intracellular domain of $\mathrm{N}$ to inhibit that cell from becoming a SMC (Lieber et al. 1992).

\section{Mosaic analysis of competition: mutual inhibition}

Mitotic recombination not only can be used to produce clones of cells that have become homozygous for a particular mutation but also to generate cells that have 
three doses instead of two, or two doses instead of one, of a particular gene. In this case, the aim of the analysis is not to assess the phenotype of the cells that have one, two, or three doses--they are usually normal-mbut to examine the interactions between cells that have different levels of one particular gene product. That is, the question is not For what is this gene required, but To what extent does the concentration of this product determine the outcome of this process?

A first set of experiments involved confronting cells with one and two, or two and three, doses of a wild-type copy of $N$. These experiments showed that whenever the boundary between haplo- and diplo- or between diploand triplo- $N$ tissue runs near the prospective position of a sense organ, the organ always derived from one of the cells with the lower dose of $N$. This result indicates that cells containing more $N$ have a lower chance of becoming a SMC, and, therefore, that the level of $N$ is one of the key elements regulating the decision to become a SMC (Heitzler and Simpson 1991).

In keeping with the idea that $D l$, and more precisely its extracellular domain, is the inhibitory signal, it was also observed that when cells with different doses of $D I$ are juxtaposed, those with a higher level of $D l$ have a better chance of becoming the SMC. Taken together, these results suggest that the lateral inhibition is mediated by a molecular interaction between $\mathrm{Dl}$ acting as an inhibitory signal and $\mathrm{N}$ acting as its receptor (Heitzler and Simpson 1991).

On the basis of these results and the role played by a structurally homologous gene acting in vulval development in the nematode Caenorhabditis elegans /Seydoux and Greenwald 1989\}, Simpson \{1990\} has proposed that lateral inhibition occurs among the cells of the proneural clusters before the singling out of the SMC. This system of inhibition is perhaps best termed "mutual inhibition" to distinguish it from the initial concept of lateral inhibition whereby an already singled out SMC inhibits its neighbors from becoming SMC (Goriely et al. 1991).

Assuming that there is some feedback mechanism by which the activation of $\mathrm{N}$ product in a cell reduces the ability of this cell to produce the $\mathrm{Dl}$ protein, the result will be a self-reinforcing effect whereby a cell with more activated $\mathrm{N}$ will produce less $\mathrm{Dl}$ and will therefore be less inhibitory to its neighbors. The neighbors, having less of their own $\mathrm{N}$ activated, will produce more $\mathrm{Dl}$ and will activate more and more $\mathrm{N}$ of the initial cell. That is, small differences in the level of activated $\mathrm{N}$ will evolve autocatalytically into large differences with time. By the same argument, cells with more doses of $D I$ will be more inhibitory to their neighbors and will therefore be less and less inhibited themselves.

By this mechanism, any small difference in the levels of active $\mathrm{Dl}$ and/or $\mathrm{N}$ products will be amplified until one cell becomes maximally inhibitory and its neighbors become maximally inhibited. An interesting aspect of this mechanism is that assuming only a completely uninhibited cell can become a SMC, only one cell in each cluster can ever become a SMC. As long as two (or more) cells remain in competition, they will keep on inhibiting each other, and, therefore, neither of the two can become a SMC. This aspect of the system is not only of academic interest: A careful examination of the emergence of SMCs using a SMC-specific enhancer trap line has revealed that two to three cells (pre-SMCs) can emerge from a cluster, although in all cases only one SMC will eventually form (Huang et al. 1991). Likewise, the analysis of the distribution of AS-C proteins showed that before one cell clearly emerges as the $\mathrm{SMC}$, as judged by its high level of AS-C expression, two or more cells can often be detected that express AS-C at a higher level than the surrounding cells (Cubas et al. 1991; Skeath and Carroll 1991|. These results indicate that these pre-SMCs keep on competing until only one is left, as would be predicted by a system of mutual inhibition.

\section{Mosaic analysis of competition: levels of competence}

Although the original proposal contemplated only the $N-D l$ interaction as the determining factor in the singling out of the SMC, it is now necessary to consider the role of the AS-C genes in this process.

A second set of mosaic experiments involved juxtaposing cells with different doses of the AS-C genes /Cubas et al. 1991|. In mosaics, where some of the cells have two doses of AS-C while others have only one, the former have a two to four times higher chance of becoming SMCs. This difference persists until the time at which the expression of AS-C is no longer required for the formation of the SMC. It follows that at the time the decision is taken, cells with more copies of AS-C, and presumably higher levels of AS-C products, have an advantage in the competition to shift from the competent to the determined stage.

The major difference between the proneural cells and the SMC is the level of expression of the AS-C genes. We have seen that this level increases abruptly in the SMC relative to the other cells of the cluster, defining a determined level as opposed to the competent level present in the cluster. It seems likely that the decision to become a SMC is directly related to the shift from lower to higher levels of AS-C expression. This shift is probably mediated by turning on the self-activating loop whereby each AS-C gene can activate its own expression (as well as the expression of the other AS-C genes). Thus, a simple device by which cells could become determined is that some threshold level of AS-C activity is required to turn on the autocatalytic regulatory site. There is evidence, at least for one of the AS-C genes, that an AS-C control sequence activates the transcription of a reporter gene only in the SMC, suggesting that this sequence responds only to high levels of AS-C activity (Martinez and Modolell 1991). Cells in which the concentration of AS-C products has not reached the threshold will remain at a competent state where the expression of AS-C depends on the original, position-dependent activators, whereas cells in which the threshold is reached will turn on the autocatalytic regulatory loop and shift to a high level of AS-C expression irrespective of the presence or absence of the original activators. It has been observed that the 
expression of AS-C in some SMCs is maintained longer than in the surrounding proneural cells from which they have arisen, in accordance with the idea that the AS-C expression in the SMC has become self-supporting. It is obvious in this system that cells with more doses of AS-C will reach the threshold sooner than the others and, therefore, will have a higher probability of being the first to shift to the determined level.

If the determining factor is the activation of the autocatalytic expression of AS-C, what matters is the level of activity of the AS-C products, relative to the threshold for autocatalysis, not simply the level of expression of the genes. Thus, by inactivating part of the AS-C products or their cofactor, the da protein, emc is modifying the threshold effectively and therefore plays an equally important role in defining which cell will finally become a SMC, as shown by LOF-GOF analysis.

\section{Reaching a decision}

Both the LOF-GOF and the mosaic analyses strongly suggest that the $N / D I$ system of cell interactions on one hand, and the AS-C/emc system of competence on the other hand, play a direct role in the final decision to single out one of the proneural cells as a sense organ precursor. How do the two systems interact? The easiest possibility is that of a direct effect of one system on the other. Thus, the activation of $N$ might result in a decrease in AS-C expression or activity in the cell. Alternatively, $N$ and/or $D 1$ might be among the targets of AS-C. It appears that both possibilities are probably exploited.

The analysis of the distribution of AS-C products in imaginal discs provides little evidence that the inhibitory mechanism acts to down-regulate the expression of the AS-C genes. It has been reported that after immunovisualization of AS-C proteins, SMCs are occasionally surrounded by a halo void of immunoreactive nuclei (Cubas et al. 1991). Although the effect may, in most cases, be the result of the enlargement of the SMCs and the subsequent exclusion of other nuclei nearby, it may also be that in some cases the transcription of AS-C is effectively reduced in neighboring cells. There does not seem to be any consistent effect, however, because in some cases the expression of AS-C is turned off first in the SMC and later in the other cells, whereas in other cases the opposite is true. Furthermore, at the crucial time when a few pre-SMCs are fighting each other, there is no evidence that the expression of AS-C in the other cells of the cluster is affected. Thus, although it cannot be ruled out that the expression of AS-C might eventually be decreased as a result of the process of inhibition, it seems unlikely that such an effect could mediate this process. There is evidence, on the other hand, that inhibition interferes with the function of the AS.C genes at the level of the proteins, either by modifying them or sequestering them, or probably by doing both.

The shaggy (sgg) gene product, which seems to be required for the process of inhibition and the coupling of the states of being more inhibited and becoming less inhibitory, is a kinase (Bourouis et al. 1990) that might well act on AS-C proteins. One anti-sc antibody seems to recognize only a phosphorylated form of the sc protein, and immunocytochemical studies using this and other anti-sc antibodies suggest that the sc protein may be present in a differently phosphorylated form in the cell that is singled out from the proneural cluster to become the neural precursor (Cabrera 1990).

Among the battery of neurogenic genes, some at the $E$ (spl) locus code for a group of $\mathrm{HLH}$ proteins. Analogous to the proposal that the active form of sc or other proneural gene products could be titrated by the emc protein, the HLH proteins produced by the $E(s p l)$ complex might conceivably inactivate the AS-C products by sequestering them. Alternatively, the $\mathrm{E}(\mathrm{spl})$ proteins could bind to cis-acting elements of target genes of AS-C products, thereby interfering with the function of AS-C (Tietze et al. 1992).

The second type of interaction between the proneural and neurogenic systems would be that the AS-C genes control part of the inhibition machinery. This would require that the inhibiting signal should be under the direct or indirect control of the AS-C genes. The pattern of expression of the gene coding for the signaling protein $\mathrm{DI}$ reveals that this gene is expressed at the same positions as the AS-C genes in most regions of the wing imaginal disc. Furthermore, the distribution of $D l$ transcripts is affected in mutants of the AS-C genes, indicating that DI is controlled by these genes (A. Ghysen and C. DamblyChaudière, unpubl.). Similar results were obtained with an enhancer trap line in which $l a c Z$ is inserted near $D l$. Here, again, the pattern of expression of lacZ is similar to that of the AS-C genes in the wing disc, and individual clusters of expression are abolished in AS-C mutants that eliminate AS-C expression at these sites (P. Heitzler, F. Huang, C. Dambly-Chaudière, A. Ghysen, and P. Simpson, in prep.). Finally, it has been shown that $N^{-}$ cells are capable of sending the inhibiting signal to neighboring wild-type cells, even if they are unable to form bristles, because they are mutant for either $a c$ or $s c$. $N^{-}$cells that are also deleted for the entire AS-C complex, on the other hand, lose the ability to inhibit neighboring cells, demonstrating that the inhibitory signal is under the control of AS-C genes (P. Heitzler, F. Huang, C. Dambly-Chaudière, A. Ghysen, and P. Simpson, in prep.).

It seems very likely, therefore, that competence and inhibition are directly coupled through this control. The more a cell becomes competent, the more it inhibits its neighbors and the less it will be inhibited by them. We conclude that the two systems, one providing competence and the other inhibition, are linked in two ways: An increase in inhibition leads to a decrease in competence (presumably by interfering with the activity of the AS-C proteins) while an increase in competence reinforces the inhibitory effect on the neighboring cells (by stimulating the expression of Dl). In this way, each cell actually controls its own expression of AS-C via a regulatory loop that runs through the surrounding cells. 


\section{Logic of choice and field properties}

The logical structure of the interaction between the proneural and the neurogenic genes is shown in Figure 2. It appears that AS-C is included in a positive feedback loop that comprises two negative elements. It has been shown that positive feedback loops (i.e., loops that contain an even number of negative controls) force each element of the system to choose between two extreme levels and are therefore essential components of stable switches (Thomas and D'Ari 1990). It is no surprise, then, that the AS-C genes should be included in a positive feedback loop: Autocatalytic properties have been demonstrated for other genes involved in defining cell fates.

The interesting feature of the loop shown in Figure 2, however, is that it runs between adjacent cells, such that the decision of each cell to adopt a basal or a maximal

Competent cells: mutual inhibition
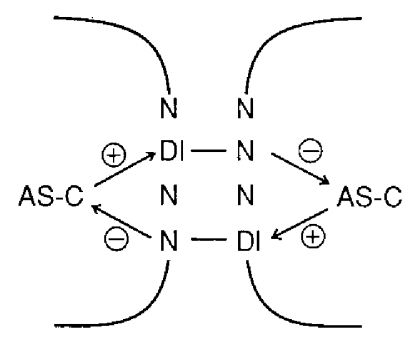

Precursor cell: lateral inhibition

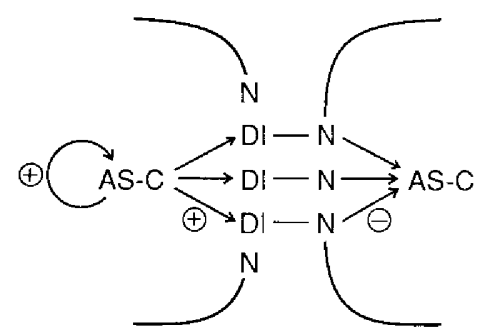

Figure 2. An intercellular positive feedback loop mediates both mutual inhibition between competent cells and lateral inhibition around a precursor cell. (Top) Within the competent field, all cells express the inhibitory signal $\mathrm{Dl}$ and receive the inhibitory signal from their neighbors, resulting in the establishment of a system of mutual inhibition. In this system the level of sc activity in one cell depends on its level in neighboring cells, and the cluster of competent cells will effectively behave as a morphogenetic field. (Bottom) As one cell in the proneural cluster becomes less inhibited, its AS-C gene activity increases, leading to further production of the inhibitory signal Dl. When its surrounding cells are all sufficiently inhibited so that they no longer generate any inhibitory signal, the absence of activated $\mathrm{N}$ product in the membrane of this cell will allow the maximal expression of AS-C by autocatalytic activation, and the morphogenetic field is then converted into a single cell that is determined as a neural precursor, surrounded by cells that are subject to lateral inhibition from the precursor. The two stages are presented as if they were distinct, but it should be noted that they are merely the two extreme stages of a progressive transition and that at most stages mutual inhibition $\mid$ top $\mid$ and autocatalytic activation of $s c$ (bottom) coexist and affect each other. level of expression of AS-C depends on the levels of activity of the AS-C genes in all of its neighbors. It follows that the control of AS-C in each individual cell is a collective, multicellular process: Integration is achieved by building cellular connectivity into the control of the key element, the level of AS-C activity. The genes involved in feeding back the activity of AS-C on itself through this intercellular loop may appear in genetic tests to be responsible for the final choice, much as the AS-C genes themselves.

Another interesting feature of the loop presented in Figure 2 is that the collective nature of the decision is limited to the cells of the proneural cluster. As far as the decision to form a SMC is concerned, the cells of a proneural cluster are effectively insulated from the rest of the tissue because the surrounding cells do not express the membrane protein that allows communication. Therefore, each proneural cluster will behave as a single morphogenetic field and will be completely unaffected by the presence or absence of additional fields in other regions of the tissue.

The example of $D l$ suggests that the existence of a field depends on establishing a system of cell communication in the appropriate set of cells. If this example is of general value, it suggests that a set of cells that behaves as a morphogenetic field for one particular developmental operation does not necessarily behave as a field for another concomitant operation. For example, cells in different regions of the notum of the fly belong to different fields as far as the formation of SMCs is concerned but probably belong to the same field as far as the control of tissue size is concerned.

A system in which cells could communicate simultaneously about different matters, or stop communicating about yet other questions, may help to reconcile two seemingly contrasting requirements of morphogenesis: the necessity of all the cells in a tissue to keep "talking" about matters of general concern, such as proliferative behavior of the tissue as a whole, and the necessity of smaller groups of cells to self-organize into local structures. The existence of dedicated communication systems would at the same time define the size and shape of the field (which will consist of the subset of cells that express the particular communication system) and the nature of the developmental process that will take place in this field. As far as this process is concerned, then, the field will behave as a completely autonomous unit.

The existence of a field may be very transient, as in the case of the proneural clusters. In this case, it is possible to use the same communication system again and again at different times of development or in different contexts, much as $N$ is involved not only in the singling out of the SMC but also in the choice of cell fate during the development of the eye facets (Cagan and Ready 1989), in the differentiation between external and internal sublineages into the formation of external sense organs (Hartenstein and Posakony 1990), in the delineation between veins and intervein regions during wing development (Diaz-Benjumea et al. 1989), in the differentiation between different muscle fibers during muscle formation 
(Corbin et al. 1991), and in the choice of different follicle cell fate during oogenesis (Ruohola et al. 1991).

Transient fields make it possible to superimpose independent patterns, because they are produced at different times of development. An example of this is the formation of two very different patterns by the same set of genes: the pattern of large bristles (each of which occupies a unique position) and the pattern of small bristles (which are regularly spaced but do not occupy fixed positions). Both patterns are formed in the same tissue (the notum) and depend on the same genetic elements: the AS-C genes $a c$ and $s c$, emc, and the neurogenic genes $N$ and $D I$. But the pattern of large bristles is completed, and the corresponding fields have disappeared several hours before the pattern of small bristles begins to form.

\section{Other patterns, other rules?}

In this review we discuss how three sets of genes work in concert to single out the precursor cells for a particular type of sense organ, the sensory bristles. Much remains to be worked out before one fully understands the interaction and regulation between these gene products. Although the same logic underlying the singling out of sensory bristles may well apply to the genesis of many other neural precursors both in the PNS and in the CNS, there are likely to be twists and surprises awaiting the analysis of these systems. For instance, AS-C genes are not required for the formation of internal sense organs, the chordotonal organs. A newly identified bHLH gene, atonal (ato), has LOF and GOF phenotypes that are qualitatively similar to those of AS-C, except that the precursors affected give rise to chordotonal organs rather than sensory bristles. Thus, ato is probably the proneural gene for chordotonal organs. The ato protein, like AS-C gene products, can form heterodimers with the da protein and bind DNA (A. Jarman, Y. Grau, L.Y. Jan, and Y.N. Jan, in prep.).

It is known that the same set of neurogenic genes involved in restricting the number of sensory bristles also controls the number of chordotonal organs. There is, however, an interesting difference between the spatial arrangement of sensory bristles and chordotonal organs. Sensory bristles (except for those at the wing margins) do not occupy the same position; in contrast, chordotonal organs tend to form bundles. Comparison of the inhibitory processes in these two cases may reveal yet unexpected features of the interplay between gene interactions and cell interactions.

\section{Acknowledgments}

A.G. thanks D. Thierry-Mieg for her inspiring hospitality. We thank Pat Simpson for sharing ideas and unpublished results. The collaborative work between the two laboratories was supported by a NATO exchange fellowship and by the Howard Hughes Medical Institute. Additional support was obtained from the Human Frontier Science Programme Organization and the Communaute Française de Belgique (to A.G. and C.D.C.). A.G. is maitre de recherches of the Fonds National de la Re- cherche Scientifique (Belgium). L.Y.J. and Y.N.J. are Howard Hughes investigators.

\section{References}

Alonso, M.C. and C.V. Cabrera. 1988. The achaete-scute gene complex of Drosophila melanogaster comprises four homologous genes. EMBO J. 7: 2585-2591.

Balcells, L., J. Modolell, and M. Ruiz-Gomez. 1988. A unitary basis for different Hairy-wing mutations of Drosophila melanogaster. EMBO I. 7: 3899-3906.

Botas, J., J. Moscoso del Prado, and A. Garcia-Bellido. 1982. Gene-dose titration analysis in the search of trans-regulatory genes in Drosophila. EMBO \%. 1: 307-310.

Bourouis, M., P. Moore, L. Ruel, Y. Grau, P. Heitzler, and P. Simpson. 1990. An early embryonic product of the gene shaggy encodes a serine/threonine protein kinease related to the CDC28/cdc2 $2^{+}$subfamily. EMBO J. 9: 2877-2884.

Cabrera, C.V. 1990. Lateral inhibition and cell fate during neurogenesis in Drosophila: The interactions between scute, Notch and Delta. Development 109: 733-742.

Cabrera, C.V. and M.C. Alonso. 1991. Transcriptional activation by heterodimers of the achaete-scute and daughterless gene products of Drosophila. EMBO J. 10: 2965-2973.

Cagan, R.L. and D.F. Ready. 1989. Notch is required for successive cell decisions in the developing Drosophila eye. Genes \& Dev. 3: 1099-1112.

Campuzano, S., L. Carramolino, C.V. Cabrera, M. Ruiz-Gomez, R. Villares, A. Boronat, and J. Modolell. 1985. Molecular genetics of the achaete-scute gene complex of $D$. melanogaster. Cell 40: 327-338.

Campuzano, S., L. Balcells, R. Villares, L. Carramolino, L. Garcia-Alonso, and J. Modolell. 1986. Excess function Hairywing mutations caused by gypsy and copia insertions within structural genes of achaete-scute complex. Cell 44: 303312.

Caudy, M., H. Vässin, M. Brand, R. Tuma, L.Y. Jan, and Y.N. Jan. 1988. daughterless, a Drosophila gene essential for both neurogenesis and sex determination has sequence similarities to myc and achaete-scute complex. Cell 55: 1061-1067.

Corbin, V., A.M. Michelson, S.M. Abmayr, V. Neel, E. Alcamo, T. Maniatis, and M.W. Young. 1991. A role for the Drosophila neurogenic genes in mesoderm differentiation. Cell 67: 311-323.

Cubas, P. and J. Modolell. 1992. The extramacrochaetae gene provides information for sensory organ patterning. EMBO $\%$ 11: 3385-3393.

Cubas, P., J.F. de Celis, S. Campuzano, and J. Modolell. 1991. Proneural clusters of achaete-scute expression and the generation of sensory organs in the Drosophila imaginal wing disc. Genes \& Dev. 5: 996-1008.

Dambly-Chaudière, C. and A. Ghysen. 1987. Independent subpatterns of sense organs require independent genes of the achaete-scute complex in Drosophila larvae. Genes \& Dev. 1: 297-306.

Diaz-Benjumea, J., M.A.F. Gonzalez Gaitan, and A. Garcia-Bellido. 1989. Developmental genetics of the wing vein pattern of Drosophila. Genome 31: 612-619.

de Celis, J.F., M. Mari-Beffa, and A. Garcia-Bellido. 1991. Cell autonomous role of the Notch gene, an epidermal growth factor homolog, in sensory organ differentiation in Drosophila. Proc. Natl. Acad. Sci. 88: 632-636.

Dietrich, U. and J.A. Campos-Ortega. 1984. The expression of neurogenic loci in imaginal epidermal cells of Drosophila melanogaster. J. Neurogenet. 1: 315-332.

Ellis, H.M., D.R. Spann, and J.W. Posakony. 1990. extramacro- 
chaetae, a negative regulator of sensory organ development in Drosophila, defines a new class of helix-loop-helix protein. Cell 61: 27-38.

Foster, G.G. 1975. Negative complementation at the Notch locus of Drosophila melanogaster. Genetics 81: 99-120.

Garcia-Alonso, L.G. and A. Garcia-Bellido. 1986. Genetic analysis of Hairy-wing mutations. WilheIm Roux's Arch Dev. Biol. 195: 259-264.

Garcia-Bellido, A. 1978. Genetic analysis of the achaete-scute system of Drosophila melanogaster. Genetics 91: 491-520.

Garcia-Bellido, A. and P. Santamaria. 1978. Developmental analysis of the achaete-scute system of Drosophila melanogaster. Genetics 88: 469-486.

Garrell, J. and J. Modolell. 1990. The Drosophila extramacrochaetae locus, an antagonist of proneural genes that, like these genes, encodes a helix-loop-helix DNA binding protein. Cell 61: 39-48.

Ghysen, A. and C. Dambly-Chaudiere. 1989. Genesis of the Drosophila peripheral nervous system. Trends Genet. 5: 251-255.

Golic, K.G. and S. Linquist. 1989. The FLP recombinase of yeast catalyzes site-specific recombination in the Drosophila genome. Cell 59: 499-509.

Gonzalez, F., S. Romani, P. Cubas, J. Modolell, and S. Campuzano. 1989. Molecular analysis of asense, a member of the achaete-scute complex of Drosophila melanogaster, and its novel role in optic lobe development. $E M B O \quad$. 8: 35533562.

Goriely, A., N. Dumont, C. Dambly-Chaudiere, and A. Ghysen. 1991. The determination of sense organs in Drosophila: Ef fect of the neurogenic mutations in the embryo. Develop ment 113: 1395-1404.

Hartenstein, V. and J.W. Posakony. 1990. A dual function of the Notch gene in Drosophila sensillum development. Dev. Biol. 142: 13-30.

Hartley, D.A., T. Xu, and S. Artavanis-Tsakonas. 1987. The embryonic expression of the Notch locus of Drosophila melanogaster and the implications of point mutations in the extracellular EGF-like domain of the predicted protein. EMBO I. 6: 3407-3417.

Hartley, D.A., A. Preiss, and S. Artavanis-Tsakonas. 1988. A deduced gene product from the Drosophila neurogenic locus Enhancer of split shows homology to mammalian G-protein $\beta$ subunit. Cell 55: 785-795.

Heitzler, P. and P. Simpson. 1991. The choice of cell fate in the epidermis of Drosophila. Cell 64: 1083-1092.

Huang, F., C. Dambly-Chaudière, and A. Ghysen. 1991. The emergence of sense organs in the wing disc of Drosophila. Development 111: 1087-1095.

Kelley, M.R., S. Kidd, W.A. Deutsch, and M.W. Young. 1987. Mutations altering the structure of epidermal growth factorlike coding sequences at the Drosophila Notch locus. Cell 51: $539-548$.

Kidd, S., M.R. Kelley, and M.W. Young. 1986. Sequence of the Notch locus of Drosophila melanogaster. Relationship of the encoded protein to mammalian clotting and growth factors. Mol. Cell. Biol. 6: 3094-3108.

Klämbt, C., E. Knust, K. Tietze, and J.A. Campos-Ortega. 1989. Closely related transcripts encoded by the neurogenic gene complex Enhancer of split of Drosophila melanogaster. EMBO I. 8: 203-210.

Kopczynski, C.C., A.K. Alton, K. Fechtel, P.J. Kooh, and M.A.T. Muskavitch. 1988. Delta, a Drosophila neurogenic gene, is transcriptionally complex and encodes a protein related to blood coagulation factors and epidermal growth factor of vertebrates. Genes \& Dev. 2: 1723-1735.
Lawrence, P.A. 1992. The making of a fly. Blackwell Scientific Publications, Oxford, UK.

Lehmann, R., U. Dietrich, F. Jiménez, and J.A. Campos-Ortega. 1981. Mutations of early neurogenesis in Drosophila. Wilhelm Roux's Arch. Dev. Biol. 190: 226-229.

Lewis, E.B. 1978. A gene complex controlling segmentation in Drosophila. Nature 276: 565-570.

Leyns, L., C. Dambly-Chaudiere, and A. Ghysen. 1989. Two different sets of cis elements regulate scute to establish two different sensory patterns. Wilhelm Roux's Arch. Dev. Biol. 198: 227-232.

Lieber, T., C.S. Wesley, E. Alcamo, B. Hassel, J.F. Krane, J.A. Campos-Ortega, and M. Young. 1992. Single amino acid substitutions in EGF-like elements of Notch and Delta modify Drosophila development and affect cell adhesion in vitro. Neuron 9: 847-859.

Martinez, C. and J. Modolell. 1991. Cross-regulatory interactions between the proneural achaete and scute genes of Drosophila. Science 251: 1485-1487.

Murre, C., P.S. McCaw, H. Vaessin, M. Caudy, L.Y. Jan, Y.N. Jan, C. Cabrera, A.B. Buskin, J.N. Hauschko, S.D. Lassar, H. Weintraub, and D. Baltimore. 1989. Interactions between heterologous helix-loop-helix proteins generate complexes that bind specifically to a common DNA sequence. Cell 58: $537-544$

Nüsslein-Volhard, C. and E. Wieschaus. 1980. Mutations affecting segment number and polarity in Drosophila. Nature 287: 795-801.

Portin, P. 1975. Allelic negative complementation at the Abruptex locus of Drosophila melanogaster. Genetics 81: 121133.

Poulson, D.F. 1937. Chromosomal deficiencies and the embryonic development of Drosophila melanogaster. Proc. Nat. Acad. Sci. 23: 133-137.

Rebay, I., R.J. Fleming, R.G. Fehon, L. Cherbas, P. Cherbas, and S. Artavanis-Tsakonis. 1991. Specific EGF repeats of Notch mediate interactions with Delta and Serrate: Implications for Notch as a multifunctional receptor. Cell 67: 687-699.

Rodriguez, I., R. Hernandez, J. Modolell, and M. Ruiz-Gomez. 1990. Competence to develop sensory organs is temporally and spatially regulated in Drosophila epidermal primordia. EMBO J. 9: 3583-3592.

Romani, S., S. Campuzano, E. Macagno, and J. Modolell. 1989. Expression of achaete and scute genes in Drosophila imaginal discs and their function in sensory organ development. Genes \& Dev. 3: 997-1007.

Ruiz-Gomez, M. and A. Ghysen. 1993. The expression and role of a proneural gene, achaete, in the development of the larval nervous system of Drosophila. EMBO I. 12: 1121-1130.

Ruiz-Gomez M., and J. Modolell. 1987. Deletion analysis of the achaete-scute locus of Drosophila melanogaster. Genes \& Dev. 1: 1238-1246.

Ruohola, H., K.A. Bremer, D. Baker, J.R. Swedlow, L.Y. Jan, and Y.N. Jan. 1991. Role of neurogenic genes in establishment of follicle cell fate and oocyte polarity during oogensis in Drosophila. Cell 66: 433-449.

St. Johnston, D. and C. Nüsslein-Volhard. 1992. The origin of pattern and polarity in the Drosophila embryo. Cell 68: 201219.

Seydoux, G. and I. Greenwald. 1989. Cell autonomy of lin-12 function in a cell fate decision in C. elegans. Cell 57: 12371245.

Simpson, P. 1990. Lateral inhibition and the development of the sensory bristles of the adult peripheral nervous system of Drosophila. Development 109: 509-519.

Skeath, J.B. and S.B. Carroll. 1991. Regulation of achaete-scute 
gene expression and sensory organ pattern formation in the Drosophila wing. Genes \& Dev. 5: 984-995.

1992. Regulation of proneural gene expression and cell fate during neuroblast segregation in the Drosophila embryo. Development 114: 939-946.

Thomas, R. and R. D'Ari. 1990. Biological feedbacks. CRC Press, Boca Raton/Ann Arbor/Boston.

Tietze, K., N. Oellers, and E. Knüst. 1992. Enhancer of $S p l i t^{D}$, a dominant mutation of Drosophila, and its use in the study of functional domain of a helix-loop-helix protein. Proc. Natl. Acad. Sci. 89: 6152-6156.

Van Doren, M., H.M. Ellis, and J.W. Posakony. 1991. The Drosophila extramacrochaetae protein antogonizes sequence-specific DNA binding by daughterless/achaete-scute protein complexes. Development 113: 245-255.

Vässin, H., K.A. Bremer, E. Knust, and J.A. Campos-Ortega. 1987. The neurogenic locus Delta of Drosophila melanogaster is expressed in neurogenic territories and encodes a putative transmembrane protein with EGF-like repeats. $E M B O$ /. 6: 3431-3440.

Villares, R. and C.V. Cabrera. 1987. The achaete-scute gene complex of $D$. melanogaster: Conserved domains in a subset of genes required for neurogenesis and their homology to myc. Cell 50: 415-424.

Wharton, K.A., K.M. Johansen, T. Xu, and S. Artavanis-Tsakonas. 1985. Nucleotide sequence from the neurogenic locus Notch implies a gene product that shares homology with proteins containing EGF-like repeats. Cell 43: 567-581.

Wigglesworth, V.B. 1940. Local and general factors in the development of "pattern" in Rhodnius prolixus (Hemiptera). $J$. Exp. Biol. 17: 180-200. 


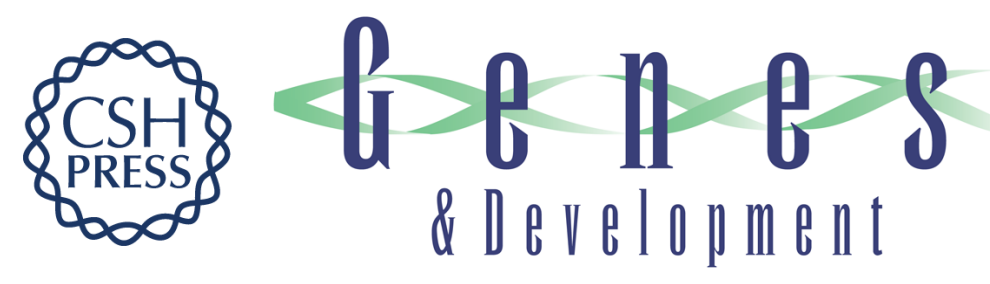

\section{Cell interactions and gene interactions in peripheral neurogenesis.}

A Ghysen, C Dambly-Chaudière, L Y Jan, et al.

Genes Dev. 1993, 7:

Access the most recent version at doi:10.1101/gad.7.5.723

References This article cites 62 articles, 21 of which can be accessed free at: http://genesdev.cshlp.org/content/7/5/723.full.html\#ref-list-1

License

Email Alerting

Receive free email alerts when new articles cite this article - sign up in the box at the Service top right corner of the article or click here.

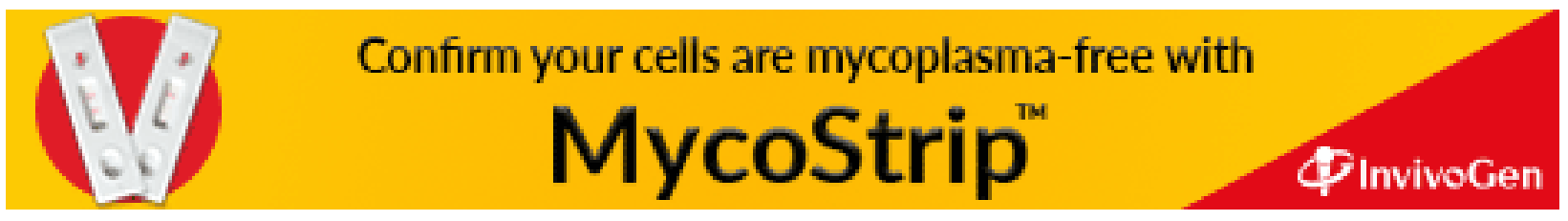

